\title{
Detection of Focal Epileptic Seizure Using NIRS Signal Based on Discrete Wavelet Transform
}

\author{
Aslan Modir ${ }^{1 *}$, Mohamad Ali Khalilzadeh ${ }^{1}$, Ali Gorji² \\ 1'Department of Biomedical Engineering, Mashhad Branch, Islamic Azad University, Mashhad, Iran \\ ${ }^{2}$ Epilepsy Research Center University of Munster, Munster, Germany
}

\begin{abstract}
Background: Despite the large number of research and significant advances in neuroscience, the hemodynamic activities of epilepsy have been rarely investigated due to high costs, need for contrast agents in FMRI and PET, lack of signals during epileptic seizure and un-portability of the equipment. Recently, Near-infrared spectroscopy (NIRS) system has attracted a large number of researchers. This system does not have the above-mentioned problems and provides a better temporal resolution than the other equipment; however, it cannot be compared to PET or fMRI, in terms of spatial resolution. The project was conducted with a feasibility study to detect epileptic seizures and extraction of epileptic dynamics using a time multiplex system at 2 wavelengths of 740 and $850 \mathrm{~nm}$. Analyzing the frequency and temporal-domains of 8 patients with focal epilepsy in temporal area during the time of sleeping, we can identify the most difference between epileptic and normal conditions in low-frequencies at the high order Daubechies wavelet transform of hemodynamic components. The main challenge is the significant resemblances between epileptic dynamic and motion artifact in low frequencies. Finally, using the most appropriate features such as Shannon entropy and the new index that we named "upgraded cumulants" showing proper separability under $t$ test and also by using different classifiers, the best result was achieved with the help of SVM classifier with an accuracy of $78.57 \%$

Keywords: Component; Detection; NIRs Signal; Epileptic Seizure; Daubechies wavelet; Upgraded cumulants; Shannon Entropy; SVM Classifier
\end{abstract}

\author{
*Correspondence to \\ Aslan Modir, Department \\ of Biomedical Engineering \\ Azad University of \\ Mashhad, Mashhad, Iran. \\ Tel: +98(51)36211865/ \\ +98(901)3359339; \\ Email: \\ aslan.modir@mshdiau.ac.ir
}

Published online October 8 2017

Citation: Modir A, Khalilzadeh MA, Gorji A. Detection of focal epileptic seizure using NIRs signal based on discrete wavelet transform. Int Clin Neurosci J. 2017;4(4):134-139. doi: 10.15171/icnj.2017.03.

\section{Introduction}

Epilepsy is a mental disorder and the earliest reports about it date back to ancient times. Instantaneous and unpredictable nervous attack in patient, vigorous shaking and seizure, uncontrollable body movements, extreme muscle contractions and/or collapse are some of the symptoms of epilepsy that vary according to the type and severity of the disease. ${ }^{1}$

Epilepsy is of the most common neurological disorders and 65000000 people in the world are affected by this disease. About 800000 of the patients are in Iran.

Prescribing the type and dosage of the drug in the long-term process of drug therapy requires monitoring parameters such as occurrence time and the number of seizures, the duration of the ictal phase in each seizure, and the severity of the seizures, which tell the importance of detecting epileptic seizures. Also, diagnostic care and minimizing damages are clear. On the other hand, with regard to the loss of epileptic hemodynamic in systems such as fMRI due to severe convulsions, the extraction of hemodynamic of epileptic seizures in this system is important in terms of studies in neuroscience branches. All of the aforementioned items represent the special place of research in this field.

Medical treatment is the most prevalent therapy method and in the cases that the patient is resistant to the drugs, surgery and removal of focal epileptic area are recommended. Unfortunately, the latter is not influential enough and the disease will return after several years. ${ }^{2}$ Therefore, mobile care systems have been used during the treatment process in order to detect the seizure moments and to predict the attacks in resistant- to-drug patients. The proposed systems are designed based on registering electrical signals of the brain. Due to extreme noises and artifacts, ECoG is used for registering the signals; the registration process of this type requires severe surgeries and installing and electrode on the brain cortex.

Near-infrared spectroscopy (NIRS) system measures the brain activity indirectly with a slow dynamic. In fact, the system can measure the local metabolism of brain cortex

(C) 2017 The Author(s). This is an open-access article distributed under the terms of the Creative Commons Attribution-NonCommercial 4.0 International License (http://creativecommons.org/licenses/by-nc/4.0/) which permits copy and redistribute the material just in noncommercial usages, provided the original work is properly cited. 
by evaluating the amount of oxygenated and withoutoxygen hemoglobin of blood in a non-invasive manner. The process is performed through modulated radiation and evaluation of reflection intensities of 2 or more optical wavelengths in an area near to infrared where the absorption coefficient of chromophore of hemoglobin is high as seen in Figure 1. Then, the absolute or approximate amounts of hemoglobin and oxyhemoglobin are calculated based on intensity of the absorbed radiation in a certain wavelength and by solving Beer-Lambert modified equation system. ${ }^{3}$

Through the current study, we have tried to indirectly measure the brain activities using NIRS -that has a simple hardware system- in order to investigate epileptic information in the signals and to detect epileptic seizures in a non-invasive manner. Doing so, we would be able to obtain the epileptic hemodynamic signal that cannot be reached in other systems due to extreme movement artifacts.

Since 2008, several papers about evaluation of epileptic seizure using NIRS signals have been written. ${ }^{4}$ Recently, a group of researchershaveused these signalsin order to detect epileptic seizure; but due to weakness of the processing method, their reports have become unsuccessful. ${ }^{5}$ What is important is that through all of the studies reported up to now, different complex-hardware registration systems such as frequency domain or time resolved have been used. ${ }^{6,7}$ These systems show high sensitivities at the time of signal registration; so that the epileptic spikes corresponding to delayed EEG signals are easily detected and observed in hemodynamic components. But these systems are big in size, expensive and hard-to-carry; so, the range of motion would become highly limited. The system used in the current study is really simple and portable. However, it is less sensitive.

\section{Materials and Methods \\ Recording System}

As mentioned before, depending on the technique applied for measurement and on the registration sensitivity, there are different registration systems that are generally heavy and expensive. These systems have complicated hardware systems, because they are going to calculate the absolute values of the parameters. However, the system used in the current study is designed to be a mobile care system; so, it is less expensive, smaller in size and less sensitive and it calculates the hemodynamic components, approximately.

In the proposed system, $740-\mathrm{nm}$ and $850-\mathrm{nm}$ wavelengths are used. The reasons for selecting these components are high molecular absorption coefficients of hemoglobin and oxyhemoglobin in these wavelengths and also, lower water and fat effects that are considered as the noises of the signal background.

Each LED optical resources are turned on and off at certain moments in order for the evaluation process not to be unwantedly interrupted.

Optical intensities in both of the transmitters are similar

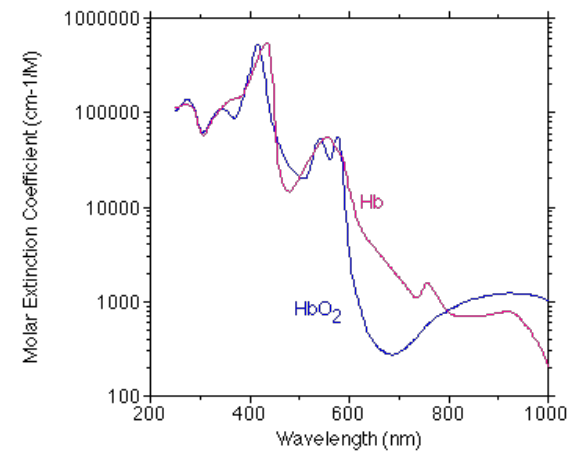

Figure 1. Molecular Absorption Coefficient for Some of the Substances Observed in Live Tissues Per Different Wavelengths. ${ }^{6}$

to each other are controlled by LED-drive.

We have used Beer-Lambert modified equation and calculated relative variations of the hemodynamic components. ${ }^{3}$

$\left|\begin{array}{l}\Delta X_{\text {oxy }} \\ \Delta X_{\text {deoxy }}\end{array}\right|=\left|\begin{array}{ll}\bar{I} \varepsilon_{\text {oxy }}\left(\lambda_{1}\right) & \bar{I} \varepsilon_{\text {deoxy }}\left(\lambda_{1}\right) \\ \bar{I} \varepsilon_{\text {oxy }}\left(\lambda_{2}\right) & \bar{I}_{\text {deoxy }}\left(\lambda_{2}\right)\end{array}\right|^{-1}\left|\begin{array}{l}\Delta A b s\left(\lambda_{1}\right) \\ \Delta A b s\left(\lambda_{2}\right)\end{array}\right|$

Where $\Delta X_{\text {oxy }}$ and $\Delta X_{\text {deoxy }}$ are the hemodynamic components of NIRs signal. Average passageway of the light beam is shown as $\bar{I}$ and is considered $3 \mathrm{~cm}$. Molecular absorption coefficient per wavelength $\lambda$, is shown by $\varepsilon$ and $\Delta A b s(\lambda)$ shows the variation of optical intensity measured per a certain wavelength in photodiode (sensor).

We used a $3 \mathrm{~W}$ LED with 2 mentioned wavelengths was used as a transmitter. The wavelength-sensitive photodiode as well as the internal preamplifier as a sensor were used. Assuming a distance of about $1.5 \mathrm{~cm}$ from the surface of the scalp to the cerebral cortex, according to the classic model of Korea, the distance between the transmitter and the receiver was $3 \mathrm{~cm}$, so that the EEG electrode was located in the middle. These components were fixed on a flexible electric fiber, then the back and around the optodes were shielded by silicon optical insulation to minimize optical noise. The duration of each cycle in Table 1 was $0.4 \mathrm{~ms}$ and the 16-bit analogue to digital unit sampled from each cycle, which was equivalent to a sampling rate of $10 \mathrm{kHz}$. The above operations were performed by STM-32 ARM microprocessor and were transmitted by the USB cable to the computer as shown in Figure 2. To minimize the effect of ambient light, in the program written under Windows with C\# programming language, the optical intensity of the off cycle subtracted from the previous on cycle, and averaged all 100 samples.

Table 1. Working Cycle

\begin{tabular}{lllll}
\hline Wavelength & \multicolumn{5}{l}{ LED condition during one registration cycle } \\
\hline $740 \mathrm{~nm}$ & ON & OFF & OFF & OFF \\
$850 \mathrm{~nm}$ & OFF & OFF & ON & OFF \\
\hline \multicolumn{4}{l}{ Working cycle of an optical transmitter used in temporal multiplex method. }
\end{tabular}




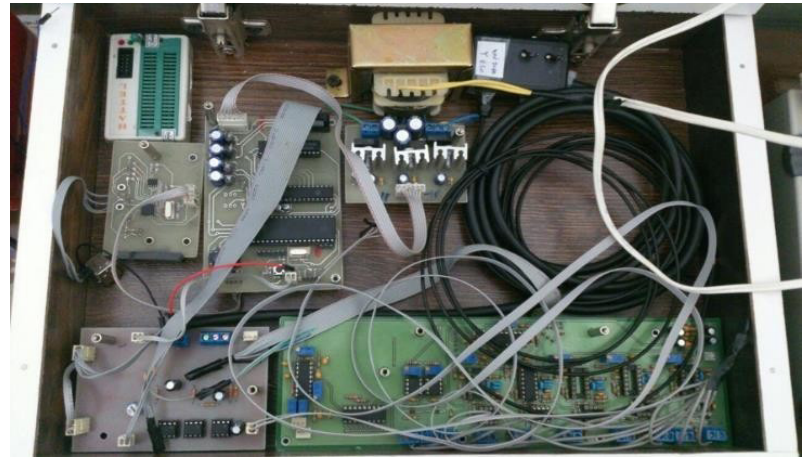

Figure 2. NIRs - EEG Recording Setup

Finally, after separating the 2 optical components and applying the Beer-Lambert law, each hemodynamic component at the rate of $25 \mathrm{~Hz}$ was displayed. The EEG registry system was connected to the main system by external trigger and the signal at the rate of $256 \mathrm{~Hz}$ was displayed simultaneously on the computer and stored in txt format. The system was validated by testing for pauses in breathing (apnea) and reducing ambient temperature, and was calibrated by a similar clinical system.

\section{Recording Protocol}

Eight patients ( 5 men and 3 women) with average age of 27 who are affected by resistant-to-drug focal epilepsy in temporal area of the brain are analyzed through the current study.

The electrode registering EEG signal is installed exactly on the focal area and both the transmitter and the receiver sensors of NIRS system are located on the 2 sides of that area in $1.5-\mathrm{cm}$ distances.

The registration process is clinically performed during the time of sleep in a dark room (without any optical noises) and the seizures are occurred in the absence of any external stimulants. In order to raise accuracy of the results, the subjects are requested to avoid drinking too much water before sleeping ${ }^{7}$; then, the accuracy of registration process would be increased and a minimum absorption coefficient will be reached. All night long, the system has been active and registered the signals. Finally, the time of seizure is detected and labeled by EEG signal.

\section{Pre-processing of NIRS Signal}

The hemodynamic components are registered by the system with a sampling frequency of $25 \mathrm{~Hz}$, while the hemodynamic information about brain activities show less-than- $1 \mathrm{~Hz}$ frequency that is near to the signal base line. On the other hand, the information about heart beat and respiratory rate suggest the frequencies of 1 and $2 \mathrm{~Hz}$, respectively. These 2 values are considered as noises of the system as seen in Figure 3. ${ }^{8}$

In order to remove the cardiorespiratory noises from the main signal, we have used a Butterworth fourth-ordered band path frequency filter with a passing frequency of

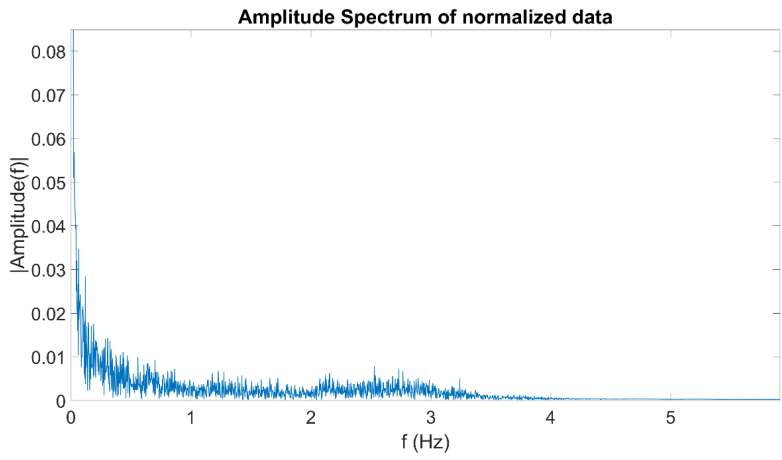

Figure 3. Frequency Components for Fourier Transformation of Oxyhemoglobin Signal. The cardiopulmonary information in the frequencies of 1 and 2 are evident.

0.01-0.3. Through most of the reference studies, the same filter has been used..$^{9,10}$ To maintain the signal variation phase and to avoid signal distortion, filtfilt command of MATLAB software has been performed.

Through the next stage, the range of signal variation has been limited between $-1 \&+1$ by normalization, because only the variation dynamic would be significant and meaningful due to the system type. So, the values are not absolute. The normalization process has been performed using equation 2 .

$N[x]=\frac{n[x]-\min \left(n_{i}\right)}{\left[\max \left(n_{i}\right)-\min \left(n_{i}\right)\right]-0.5} * 2 ; i=1$ : end

\section{Main Processing}

Since different quantitative studies are conducted on this signals in terms of engineering and analytical aspects, the analyses performed through the current research are investigated based on temporal, frequency, temporalfrequency and phase-analysis perspective. In order to maintain brevity, we would discuss some, not all, of the abovementioned perspectives.

Initially, the extracted features are statistically investigated by $t$ test and the significant differences between normal and artifact-lacking conditions of epileptic dynamics are evaluated. The 2 conditions are easily distinguishable due to their significant differences. In order to operationalize the system, we need to consider the movement artifacts that are near to the dynamics of epileptic seizure. Investigating and improving the signal properties, we can distinguish the movement artifacts and normal condition from epileptic seizure.

\section{Correlation Between Hemodynamic Components}

The correlation for the 2 components is calculated using the Process toolbox of MATLAB software, in order for the correlation analysis to be investigated deeper. In Figure 4, the horizontal time axis shows 2 signals and the vertical axis suggests positive and negative lags. Moreover, the value of correlation is specified using color indices. In lower lags that are near to zero, we see an increase in the 

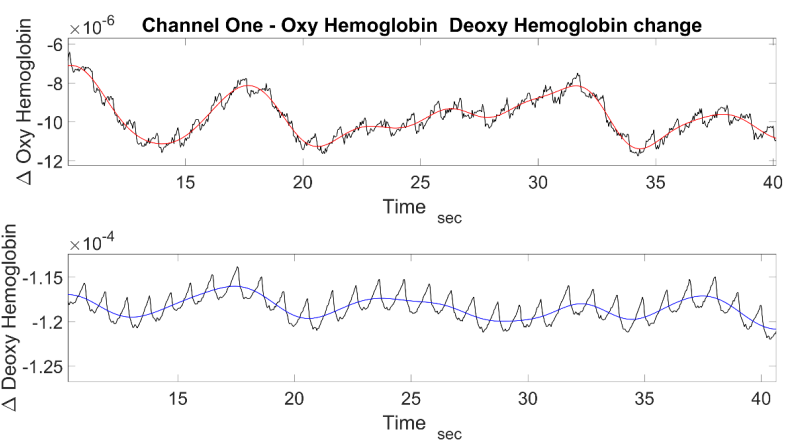

Figure 4. A Comparative Figure for Variations of 2 Hemodynamic Components of NIRS Signal Before and After Filtering.

value of correlation between the 2 components in epileptic area; this part is show with blue color in the figure. But there are other significant variations in the correlations of other lags on the time line.

\section{Short Time Fourier Transform}

Practically, when calculating Fourier transmission at the beginning and end of the numerical series, we will face some limitations due to temporal finiteness of the signal.

This limitation would be removed by adding zero to the beginning and end of the numerical series. Finiteness of temporal series, will result in an error in Fourier transmission calculations and in frequency-component extraction. In order to resolve the problem, we would use Hamming Window. To do so, we consider a sliding part with the length of 2500 samples.

The reasons for selecting such a value for the length are low cutting frequency of the filter and signal sampling rate; therefore, all of the frequency components would be present through the sliding window and high frequency resolution would be reached. Albeit, these advantages are obtained at the cost of losing temporal resolution. After selecting the part length, Hamming Window is generated according that length.

$\omega(\mathrm{n})=0.54-0.46 * \cos \left\{\frac{2 \omega n}{n-1}\right\} ; 0 \leq n \leq N-1$

But reduction in numerical range at the beginning and end will result in error. Hence, we have considered 50\% overlapping and applied the window for the whole length of the data. Then, Fourier transmission will be calculated for each window and finally, the set of frequency components of the windows will be sampled.

In order to analyze the data using MATLAB software. As seen in Figure 5, the amplitude of time-frequency segment, in low frequency components in epileptic moments has less intensity than the other components in other moments as seen in Figure 6. But the proposed method has serious bugs due to different reasons. First, according to principle of Heisenberg uncertainty, there is always a compromise between temporal resolution and frequency resolution that means an inability in

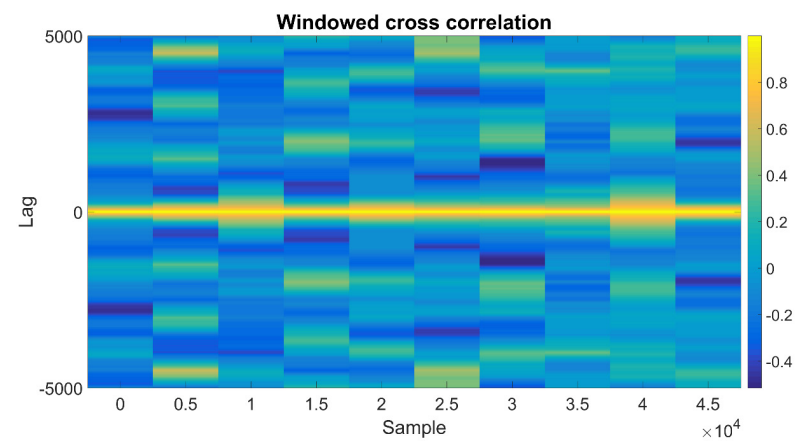

Figure 5. The Correlation Scope for Subject 1 in a Time-Line.

simultaneous mapping of temporal domain and frequency domain. Second, Fourier transmission of the correlation function would be necessarily performed and merely the information located in correlation series are included. Therefore, regarding accessibility of the most proper data of low-frequency, we can perform a multi-resolution analysis using wavelet transform.

\section{Wavelet Transform}

In a research, NIRs signal has been used to detect epilepsy seizure in mind-computer interface system. ${ }^{10,11}$ Investigating different bases, the researchers have selected base of Mother Daubechies Violet Transformation for extracting the features from the signal. We have also obtained the best results from the same base and used that for evaluating the variations of temporal-frequency scope of the signal. The relation for discrete violet transformation for Mother Violet Orthogonal Base $\Psi(t)$ is as follows:

$\mathrm{S}(\mathrm{t})=\sum_{n=-\infty}^{\infty} A_{m_{0} . n} \emptyset_{m_{0} . n}(t)+\sum_{m=-\infty}^{m_{0}} \sum_{n=-\infty}^{\infty} D_{m . n} \Psi_{m . n}(t)$

where $\emptyset_{m . n}(t)$ shows the scale function that is known as Father Function and is defined as follows:

$\emptyset_{m . n}(t)=2^{-\frac{m}{2}} \emptyset\left(2^{-m} t-n\right)$

The coefficients for major and minor components are:

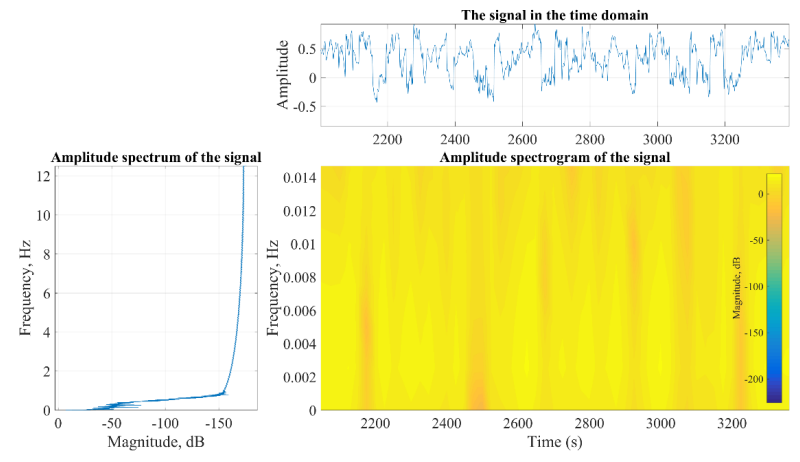

Figure 6. STFT of Oxyhemoglobin in Low Frequency Range. 


$$
\begin{aligned}
& A_{m \cdot n}=\int_{-\infty}^{\infty} S(t) \emptyset_{m \cdot n}(t) d t \\
& D_{m \cdot n}=\int_{-\infty}^{\infty} S(t) \Psi_{m \cdot n}(t) d t
\end{aligned}
$$

Through the calculations, a relatively high order (7) is selected for Vanishing Moment index in Daubechies base transformation function.

According to the sampling frequency and the range of passing frequency, all the signals are shown in Approximation part of wavelet transformation until the fifth level. In the 10th level, especially at the Approximation unit containing a great amount of information about lowfrequency signals, some of the signals that show epileptic seizure have more intense variation ranges than the surrounding areas as seen in Figure 7.

\section{Feature Extraction}

The features are extracted in order to find significant differences between seizure state and normal moments before seizure. Classic statistical properties such as average, variance, domain estimation, root mean square (RMS) and minimal and maximal domains in processing window can help us with calculating the difference. The best results are obtained at the 10th level of wavelet transform. However, in order to differentiate epileptic seizure detection from movement artifacts that have similar dynamics to epilepsy symptoms, we need more powerful features. Among many features investigated, the most effective ones are as follows.

\section{Shannon Entropy}

According to the principle of information theory, the value of distribution irregularity is used to quantify the results; and the possibility of numerical series is calculated using density distribution integral.

$$
\mathrm{S}=-K_{b} \sum p_{i} \log _{2}\left(p_{i}\right)
$$

The symbol $\mathrm{K}_{\mathrm{b}}$ shows Boltzman's constant value.

\section{Simple and Upgraded cumulants}

High-order statistical figures suggest temporal range features and include the concepts of signal morphology; these concepts are not accessible in traditional analysis methods. The generalization moments show the autocorrelation index and a non-linear combination of them is called "Cumulants". The requirements for using the moments and cumulants are static real signals and a domain distribution with average of zero. Mathematically, we can state that:

$$
\begin{aligned}
& R_{x}(\tau)=C_{2}^{x}(\tau)=E\{x(n) x(n+\tau)\} \\
& C_{3}^{x}\left(\tau_{1} \cdot \tau_{2}\right)=E\left\{x(n) x\left(n+\tau_{1}\right) x\left(n+\tau_{2}\right)\right\}
\end{aligned}
$$

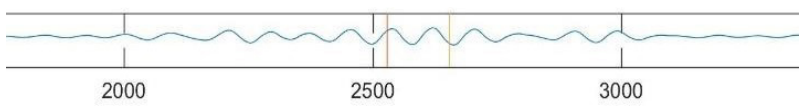

Figure 7. An Overview of Transformation Process. The 10th level of wavelet transform. The times of initiation and termination of epileptic seizure are shown with parallel vertical lines.

Equation (9) shows a second order cumulant that corresponds to the correlation in lag $\tau$. If we extend the time delay, we will be able to generate higherorder cumulants. According to the proper response of autocorrelation index to time delays approximating zero, we give a zero value to $\tau$, too. In this case, the secondto forth-order cumulants are called VAR, SKW and KRT. Statistically, these parameters show domain distribution condition, symmetry of distribution form and narrowness of signal distribution domain.

In order to extract the common features from the 2 hemodynamic components, we need an index that can use the information of both signals simultaneously. Based on the effectiveness of high-order cumulants in zero time-delay, we upgrade the cumulants or use correlation generalization for the 2 components in zero time-delay instead of generalizing autocorrelation index. According the fact that mathematical figures can be displaced, 2 and three conditions are considered for second order and upgraded fourth order cumulants, respectively.

Equations (11) and (12) are different forms of upgraded third order cumulants.

$$
\begin{aligned}
& C_{3}^{x \cdot y}(0)=E\{x(n) y(n) x(n)\} \\
& C_{3}^{x \cdot y}(0)=E\{x(n) y(n) y(n)\}
\end{aligned}
$$

\section{Statistical Evaluation}

In order to evaluate the effectiveness of the extracted feature for normal, artifact and epileptic conditions, we use $t$ test that determines the significant differences of the classes with a number between $0-10$. Zero is the ideal number; but $P$ values lesser that 0.05 also can be considered as the desired results. The requirement for performing $t$ test is normality of domain distribution. The requirements have been analyzed and confirmed using KolmogorovSmirnov test.

Simple third and fourth order cumulants, all upgraded cumulants on the 9th and 10th level of wavelet transformation of hemodynamic components and also Shannon entropy for the 10th level of wavelet transformation of oxy-hemoglobin, all have less-than-0.05 $P$ values.

\section{Detection}

In order to detect and classify three groups automatically and online using the features selected through the previous stage, we have selected three classifiers including LDA, neural network (feed forwards back propagation) 
and SVM. Among the feature vectors extracted from processing windows with $50 \%$ overlapping through epileptic, artifact and normal blocks, about $75 \%$ of the vectors are randomly selected for training and the remaining are allocated to testing. The average for highest rate of accuracy in detection after 10 times is $78.57 \%$ that belongs to SVM. Then, LDA with $75.71 \%$ accuracy rate and Neural Network with $69.99 \%$ accuracy rate take the other positions, respectively.

\section{Results and Discussion}

Heretofore, there had been no reports presented about existence of epileptic information in time-multiplex system- based NIRS signals and all of the previous researchers had utilized expensive complicated systems. ${ }^{11}$ Although the epileptic corresponding spikes of EEG signal are hardly observed in NIRS signals in the current study, the dynamic presence of epileptic seizure in the registered signal has been proven. In other systems such as fMRI, it is impossible to register the hemodynamic activities of the brain.

Previously, a group of researchers conducted a study with an aim to detect epileptic seizure using NIRS; but due to selecting inappropriate feature and also to missing signal dynamics in down sampling for reaching low frequencies, they could not reach proper-accuracy results and they claimed that the signal is inefficient in detecting the seizure. It is worth noting that these researchers used a powerful system that could calculate and show absolute values. $^{5}$

The biggest problem that make daily application of the system difficult is the presence of optical artifacts in surrounding areas. ${ }^{11}$ A great portion of these artifacts pass through the skull bone and influence on optical receiver or saturate the amplifier. Moreover, hairs can make a difficulty when using this system; because hairs, especially dark hairs, severely absorb light beams. ${ }^{12,13}$ This difficult is more intense in Iran.

A great number of research groups in different parts of the world are trying to overcome these problems and to upgrade the hardware system.

Conflict of Interest Disclosures

The authors declare that they have no conflict of interests.

Ethical Statement

All patients participating in this research have received written consent. The need for signal recording in this research is also explained to them.

References

1. Saraceno B, Avanzini G, Lee P. Epilepsy Care in the World. Geneva: World Health Organization; 2013.

2. Magiorkinis E, Sidiropoulou K, Diamantis A. Hallmarks in the history of epilepsy: epilepsy in antiquity. Epilepsy Behav. 2010;17(1):103-8. doi: 10.1016/j.yebeh.2009.10.023.

3. Jöbsis FF. Noninvasive, infrared monitoring of cerebral and myocardial oxygen sufficiency and circulatory parameters. Science. 1977;198(4323):1264-7.

4. Nishihara K, Kawai H, Gomi T, Terajima M, Chiba Y. Investigation of optimum electrode locations by using an automatized surface electromyography analysis technique. IEEE Trans Biomed Eng. 2008;55(2 Pt 1):636-42.

5. Jeppesen J. Near infrared spectroscopy as a seizure detection technology for patients with epilepsy. Clin Neurophysiol. 2016; 128(3):e101. doi:10.1016/j.clinph.2015.11.341

6. Hueber DM, Franceschini MA, Ma HY, Zhang Q, Ballesteros $J R$, Fantini S, et al. Non-invasive and quantitative near-infrared haemoglobin spectrometry in the piglet brain during hypoxic stress, using a frequency-domain multidistance instrument. Phys Med Biol. 2001;46(1):41-62.

7. Torricelli A, Contini D, Pifferi A, Caffini M, Re R, Zucchelli L, Spinelli L. Time domain functional NIRS imaging for human brain mapping. Neuroimage. 2014;85 Pt 1:28-50. doi: 10.1016/j.neuroimage.2013.05.106.

8. Cui X, Bray S, Reiss AL. Functional near infrared spectroscopy (NIRS) signal improvement based on negative correlation between oxygenated and deoxygenated hemoglobin dynamics. Neuroimage. 2010;49(4):3039-46. doi: 10.1016/j. neuroimage.2009.11.050.

9. Venclove S, Daktariunas A, Ruksenas O. Functional nearinfrared spectroscopy: a continuous wave type based system for human frontal lobe studies. EXCLI J. 2015;14:1145-52. doi: 10.17179/excli2015-614.

10. Roche-Labarbe N, Wallois F, Ponchel E, Kongolo G, Grebe R. Coupled oxygenation oscillation measured by NIRS and intermittent cerebral activation on EEG in premature infants. Neuroimage. 2007;36(3):718-27.

11. Tomita Y, Vialatte FB, Dreyfus G, Mitsukura Y, Bakardjian H, Cichocki A. Bimodal BCl using simultaneously NIRS and EEG. IEEE Trans Biomed Eng. 2014;61(4):1274-84.

12. Wallois F, Patil A, Héberlé C, Grebe R. EEG-NIRS in epilepsy in children and neonates. Neurophysiol Clin. 2010;40(56):281-92. doi: 10.1016/j.neucli.2010.08.004.

13. Sawan M, Salam MT, Gelinas S, Lan JL, Lesage F, Nguyen DK. Combined NIRS-EEG remote recordings for epilepsy and stroke real-time monitoring. Paper presented at: 2012 IEEE International Symposium on Circuits and Systems; 20-23 May 2012. 